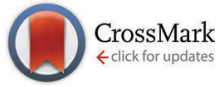

Cite this: J. Mater. Chem. C, 2016, 4, 2319

Received 23rd November 2015, Accepted 22nd February 2016

DOI: $10.1039 / c 5 t c 03930 d$

www.rsc.org/MaterialsC

\section{Chemical vapor deposition of ruthenium-based layers by a single-source approach $\dagger$}

\author{
Janine Jeschke, ${ }^{a}$ Stefan Möckel, ${ }^{a}$ Marcus Korb, ${ }^{a}$ Tobias Rüffer, ${ }^{a}$ Khaybar Assim, ${ }^{a}$ \\ Marcel Melzer, ${ }^{\text {bc }}$ Gordon Herwig, ${ }^{d}$ Colin Georgi, ${ }^{\text {bc }}$ Stefan E. Schulz ${ }^{\text {bc }}$ and \\ Heinrich Lang*a
}

A series of ruthenium complexes of the general type $\mathrm{Ru}(\mathrm{CO})_{2}\left(\mathrm{P}(n-\mathrm{Bu})_{3}\right)_{2}\left(\mathrm{O}_{2} \mathrm{CR}\right)_{2}(\mathbf{4 a}, \mathrm{R}=\mathrm{Me} ; \mathbf{4 b}, \mathrm{R}=\mathrm{Et}$; 4c, $\mathrm{R}=\mathrm{i}-\mathrm{Pr} ; \mathbf{4 d}, \mathrm{R}=t-\mathrm{Bu} ; \mathbf{4 e}, \mathrm{R}=\mathrm{CH}_{2} \mathrm{OCH}_{3} ; \mathbf{4 f}, \mathrm{R}=\mathrm{CF}_{3} ; \mathbf{4 g}, \mathrm{R}=\mathrm{CF}_{2} \mathrm{CF}_{3}$ ) was synthesized by a singlestep reaction of $\mathrm{Ru}_{3}(\mathrm{CO})_{12}$ with $\mathrm{P}(n-\mathrm{Bu})_{3}$ and the respective carboxylic acid. The molecular structures of $\mathbf{4 b}, \mathbf{4 c}$ and $\mathbf{4 e - g}$ in the solid state are discussed. All ruthenium complexes are stable against air and moisture and possess low melting points. The physical properties including the vapor pressure can be adjusted by modification of the carboxylate ligands. The chemical vapor deposition of ruthenium precursors 4a-f was carried out in a vertical cold-wall CVD reactor at substrate temperatures between 350 and $400{ }^{\circ} \mathrm{C}$ in a nitrogen atmosphere. These experiments show that all precursors are well suited for the deposition of phosphorus-doped ruthenium layers without addition of any reactive gas or an additional phosphorus source. In the films, phosphorus contents between 11 and 16 mol\% were determined by XPS analysis. The obtained layers possess thicknesses between 25 and $65 \mathrm{~nm}$ and are highly conformal and dense as proven by SEM and AFM studies.

\section{Introduction}

The ongoing miniaturization of devices in semiconductor industry has introduced new manufacturing and materials challenges. ${ }^{1}$ A promising candidate which can replace current materials in many microelectronic applications in future technology nodes is ruthenium, as it possesses a high thermal and chemical stability, a low electrical resistance and a negligible solid solubility with copper. ${ }^{1,2}$ Thin films of ruthenium are of particular interest as diffusion barriers for seedless copper interconnects in the manufacturing of integrated circuits by the damascene process. ${ }^{3-7}$ Thereby, one key destination is the development of a single material liner for future copper interconnects. However, pure ruthenium layers cannot act as sole

\footnotetext{
${ }^{a}$ Technische Universität Chemnitz, Faculty of Natural Sciences, Institute of Chemistry, Inorganic Chemistry, 09107 Chemnitz, Germany. E-mail: heinrich.lang@chemie.tu-chemnitz.de; Fax: +49(0)371-531-21219; Tel: $+49(0) 371-531-21210$

${ }^{b}$ Fraunhofer Institute for Electronic Nano Systems (ENAS), Technologie-Campus 3, 09126 Chemnitz, Germany

${ }^{c}$ Technische Universität Chemnitz, Center for Microtechnologies, 09107 Chemnitz, Germany

${ }^{d}$ Technische Universität Chemnitz, Faculty of Natural Sciences, Institute of Physics, Chemical Physics, 09107 Chemnitz, Germany

$\dagger$ Electronic supplementary information (ESI) available: Additional SEM, EDX, XPS and XRPD data. Crystallographic data of $\mathbf{4 b , c}$ and 4 e,f. CCDC 1430280 (4b), 1430281 (4c), 1430282 (4e), 1430283 (4f) and 1430284 (4g). For ESI and crystallographic data in CIF or other electronic format see DOI: 10.1039/c5tc03930d
}

diffusion barriers for copper. ${ }^{8-10}$ Due to its high surface energy, ruthenium follows a 3D (Volmer-Weber) growth mechanism and hence leads to the formation of polycrystalline, columnar films. ${ }^{11}$ These polycrystalline structures allow copper diffusion at unacceptably low temperatures through the grain boundaries. ${ }^{12,13}$ For this reason the need for the development of nanocrystalline or amorphous ruthenium-based films arises, e.g. obtained by incorporation of phosphorus. ${ }^{14,15}$ Such layers have shown to provide better copper diffusion barrier properties than pure polycrystalline ruthenium coatings. ${ }^{16}$

To date, only few approaches for the deposition of $\mathrm{Ru}(\mathrm{P})$ layers by chemical vapor deposition (CVD) or atomic layer deposition (ALD) have been described. ${ }^{11,16-19}$ Most commonly, $\mathrm{Ru}_{3}(\mathrm{CO})_{12}$ and a phosphine $\mathrm{PR}_{3}(\mathrm{R}=\mathrm{H}, \mathrm{Me}, \mathrm{Ph})$ are deposited simultaneously forming a $\mathrm{Ru}(\mathrm{P})$ layer. ${ }^{15-18}$ In this dual-source approach, the films contain $10-50 \mathrm{~mol} \% \mathrm{C}$ with decreasing $\mathrm{C}$ contents in the order $\mathrm{PPh}_{3}>\mathrm{PMe}_{3}>\mathrm{PH}_{3}{ }^{13,15,17,18}$ Furthermore, precise control and reproducibility of the film stoichiometry are difficult to achieve, because of the different vapor pressures and reactivities of both components. ${ }^{18}$ Much better reproducibilities are obtained in the single-source approach that uses precursors containing both elements in the same molecule. Moreover, a better homogeneity is provided as the desired elements are premixed at the molecular level. ${ }^{20}$ The only single-source precursor that has been examined in the deposition of $\mathrm{Ru}(\mathrm{P})$ films so far is air-sensitive $\mathrm{RuH}_{2}\left(\mathrm{PMe}_{3}\right)_{4} \cdot{ }^{11,16}$ Therein, the undesired incorporation of $\mathrm{C}$ still results in a significant increase of the film resistivity 
and reduces its applicability as a directly plateable diffusion barrier for $\mathrm{Cu}^{13}$ The quality of the films deposited from $\mathrm{RuH}_{2}\left(\mathrm{PMe}_{3}\right)_{4}$ can be improved by the addition of $\mathrm{H}_{2}$ as a reactive gas. ${ }^{15}$

We herein present the synthesis of ruthenium precursors of the general type $\mathrm{Ru}(\mathrm{CO})_{2}\left(\mathrm{P}(n-\mathrm{Bu})_{3}\right)_{2}\left(\mathrm{O}_{2} \mathrm{CR}\right)_{2}(\mathrm{R}=\mathrm{Me}$, Et, i-Pr, $t$ - $\left.\mathrm{Bu}, \mathrm{CH}_{2} \mathrm{OCH}_{3}, \mathrm{CF}_{3}, \mathrm{CF}_{2} \mathrm{CF}_{3}\right)$ and their use as single-source CVD precursors for the preparation of thin and conformal phosphorus-doped ruthenium layers. ${ }^{21}$ The influence of the carboxylate ligands on the thermal behavior and vapor pressure is discussed. The layers obtained were characterized by SEM, EDX, AFM and XPS measurements.

\section{Experimental section}

\section{Instruments and materials}

All synthesis procedures were performed under an atmosphere of argon with the solvents degassed prior to use. All reagents were obtained from commercial suppliers and used without further purification. For column chromatography, silica with a particle size of 40-60 $\mu \mathrm{m}$ (230-400 mesh (ASTM), Fa. MachereyNagel) was used.

NMR spectra were recorded using a Bruker Avance III 500 spectrometer operating at $500.3 \mathrm{MHz}$ for ${ }^{1} \mathrm{H}, 125.7 \mathrm{MHz}$ for ${ }^{13} \mathrm{C}\left\{{ }^{1} \mathrm{H}\right\}$ and $202.5 \mathrm{MHz}$ for ${ }^{31} \mathrm{P}\left\{{ }^{1} \mathrm{H}\right\}$ in the Fourier transform mode at $298 \mathrm{~K}$. Chemical shifts are reported in $\delta$ (ppm) downfield from tetramethylsilane with the solvent as a reference signal $\left({ }^{1} \mathrm{H}\right.$ NMR, $\mathrm{CHCl}_{3} \delta 7.26 ;{ }^{13} \mathrm{C}\left\{{ }^{1} \mathrm{H}\right\}$ NMR, $\mathrm{CDCl}_{3} \delta$ 77.16; ${ }^{31} \mathrm{P}\left\{{ }^{1} \mathrm{H}\right\}$ NMR, standard external relative to $\left.85 \% \mathrm{H}_{3} \mathrm{PO}_{4} \delta 0.0\right)$. FT-IR spectra were recorded using a Thermo Nicolet IR 200 instrument. The melting points were determined using a Gallenkamp MFB 595010 M melting point apparatus. Elemental analyses were performed using a Thermo FlashAE 1112 instrument. Highresolution mass spectra were recorded using a Bruker Daltonite micrOTOF-QII spectrometer using electro-spray ionization (ESI $\dagger$ ).

TG experiments were performed using a Mettler Toledo TGA/DSC1 1100 system with a UMX1 balance. CVD experiments were carried out using a home-built vertical cold-wall CVD reactor with a heater dimension of $20 \times 20 \mathrm{~mm}$ (BACH Resistor Ceramics $\mathrm{GmbH}$ ). Heating could be adjusted up to $773 \mathrm{~K}$ and was controlled by a Gefran 600 module connected with a Pt100 thermosensor. The carrier gas $\left(\mathrm{N}_{2}\right)$ was controlled by MKS type 247 mass flow controllers connected to the reactor by heated copper lines. The CVD system was attached to a rotary vane pump RZ 6 (Vacuubrand). The pressure of the reactor system was controlled by a Vacuubrand vacuum controller (CVC 3000) in combination with an external Pirani vacuum sensor (VSP 3000).

The surface morphology was investigated by field-emission scanning electron microscopy using a ZEISS Supra60 SEM. Cross-sectional SEM investigations were carried out to determine the film thickness. All AFM measurements were accomplished using NanoWizard I and II devices (JPK Instruments AG, Berlin, Germany) under ambient conditions using JPK SPM Control Software provided by the manufacturer. RMS values were determined using JPK Image Processing. The measurements were carried out in tapping mode using silicon AFM tips with a cantilever length of $125 \mu \mathrm{m}$ and a spring constant of approximately $42 \mathrm{~N} \mathrm{~m}^{-1}$ (Pointprobe ${ }^{\circledR}$ NCH, NanoWorld AG, Neuchâtel, Switzerland). Energy-dispersive X-ray analysis using a Bruker Quantax 400 system attached to a SEM was applied to determine the chemical composition of the films. The composition of the $\mathrm{Ru}$ samples was investigated using a PREVAC XPS system. Monochromatic aluminum $\mathrm{K} \alpha$ radiation $(1486.6 \mathrm{eV}$ ) was provided by a VG Scienta MX 650 X-ray source and a monochromator. The energy distribution of the photoelectrons was measured using a VG Scienta EW3000 XPS/UPS/ARPES analyzer. This analyzer was operated at $200 \mathrm{eV}$ pass energy with a step size of $100 \mathrm{meV}$ and a measurement time of $3.0 \mathrm{~s}$ for each data point. Casa XPS 2.3.16 Pre-rel 1.4 software was used for the deconvolution of the XPS peaks. For the calculation of the atomic concentration, Scofield relative sensitivity factors (RSFs) were used. These RSFs were corrected for a monochromator-analyzer angle of $52.55^{\circ}$. For the escape depth correction in Casa XPS, a value of -0.7 was applied. XRPD patterns were collected on a STOE-STADI P diffractometer using $\mathrm{Cu} \mathrm{K}_{\alpha}(1.5405 \AA)$ radiation and a $\mathrm{Ge}(111)$ monochromator.

Diffraction data were collected with an Oxford Gemini S diffractometer using graphite-monochromated Mo $\mathrm{K}_{\alpha}$ radiation $(\mathbf{4 b}, \mathbf{c}, \mathbf{f}, \mathbf{g})(\lambda=0.71073 \AA)$ or $\mathrm{Cu} \mathrm{K}_{\alpha}$ radiation $(4 \mathrm{e})(\lambda=1.54184 \AA)$ at $110 \mathrm{~K}$ using oil-coated shock-cooled crystals. The structures were solved by direct methods and refined by full-matrix leastsquares procedures on $F^{2}{ }^{22,23}$ All non-hydrogen atoms were refined anisotropically, and a riding model was employed in the refinement of the hydrogen atom positions. Graphics of the molecular structures were created by using SHELXTL $^{23}$ and ORTEP. $^{24}$

\section{Precursor synthesis and characterization}

General synthesis procedure for ruthenium complexes $4 \mathrm{a}-\mathrm{g}$. A solution of $\mathrm{Ru}_{3}(\mathrm{CO})_{12}(200 \mathrm{mg}, 313 \mu \mathrm{mol})$ and $\mathrm{P}(n-\mathrm{Bu})_{3}(738 \mathrm{mg}$, $3.65 \mathrm{mmol})$ in 4-methylpentan-2-one $(18 \mathrm{~mL})$ was refluxed for $8 \mathrm{~h}$. Subsequently, the respective carboxylic acid $(2.50 \mathrm{mmol})$ was added and the solution refluxed for further $6 \mathrm{~h}$. After solvent removal in vacuum the residue was subjected to silica-gel column chromatography (dimension $3 \times 20 \mathrm{~cm}$ ) or purified by recrystallization from $n$-pentane at $-78{ }^{\circ} \mathrm{C}$.

Synthesis of $\mathrm{Ru}(\mathrm{CO})_{2}\left(\mathbf{P}(n-\mathrm{Bu})_{3}\right)_{2}\left(\mathrm{O}_{2} \mathrm{CCH}_{3}\right)_{2}$ (4a). The title compound was synthesized according to the general procedure by using acetic acid $(150 \mathrm{mg}, 2.50 \mathrm{mmol})$. The raw product was recrystallized from $n$-pentane at $-78{ }^{\circ} \mathrm{C}$ to give the respective product as a colorless, crystalline solid. Yield: $494 \mathrm{mg}$ (727 $\mu \mathrm{mol}, 78 \%)$.

M.p. $108{ }^{\circ} \mathrm{C}$; anal. calcd for $\mathrm{C}_{30} \mathrm{H}_{60} \mathrm{O}_{6} \mathrm{P}_{2} \mathrm{Ru}$ : C, 53.00; H, 8.90; found: C, 53.07, H, 8.96; IR data $\left(\mathrm{KBr}, \nu / \mathrm{cm}^{-1}\right): 2961 \mathrm{~m}, 2930 \mathrm{~m}$, 2874 w, 2863 w, 2036 vs, 1970 vs, 1621 s, 1467 w, 1460 w, 1420 w, 1368 m, 1321 s, 1214 w, 1183 w, 1092 w, 1055 w, 1009 w, 967 w, 907 w, 895 w, 867 w, 806 w, 774 w, 746 w, 721 w, 683 w, 669 w, $625 \mathrm{w}, 602 \mathrm{~m} ;{ }^{1} \mathrm{H}-\mathrm{NMR}\left(500 \mathrm{MHz}, \mathrm{CDCl}_{3}, \delta\right): 0.92\left(\mathrm{t},{ }^{3} J_{\mathrm{HH}}=7.2 \mathrm{~Hz}\right.$, $\left.18 \mathrm{H}, \mathrm{CH}_{2} \mathrm{CH}_{3}\right), 1.33-1.50\left(\mathrm{~m}, 24 \mathrm{H}, \mathrm{PCH}_{2} \mathrm{CH}_{2}, \mathrm{CH}_{2} \mathrm{CH}_{3}\right), 1.77-1.86$ $\left(\mathrm{m}, 12 \mathrm{H}, \mathrm{PCH} \mathrm{H}_{2}\right), 1.98\left(\mathrm{~s}, 6 \mathrm{H}, \mathrm{CH}_{3}\right) ;{ }^{13} \mathrm{C}-\mathrm{NMR}\left(126 \mathrm{MHz}, \mathrm{CDCl}_{3}, \delta\right)$ : $13.8\left(\mathrm{~s}, \mathrm{CH}_{2} \mathrm{CH}_{3}\right), 23.5\left(\mathrm{t}, J_{\mathrm{CP}}=12.9 \mathrm{~Hz}, \mathrm{PCH}_{2}\right), 23.6\left(\mathrm{~s}, \mathrm{CH}_{3}\right)$, $24.5\left(\mathrm{t}, J_{\mathrm{CP}}=6.5 \mathrm{~Hz}, \mathrm{CH}_{2} \mathrm{CH}_{3}\right), 25.1\left(\mathrm{~s}, \mathrm{PCH}_{2} \mathrm{CH}_{2}\right), 177.0\left(\mathrm{~s}, \mathrm{O}_{2} C\right)$, $198.0\left(\mathrm{t}, J_{\mathrm{CP}}=11.5 \mathrm{~Hz}, C \mathrm{O}\right) ;{ }^{31} \mathrm{P}-\mathrm{NMR}\left(203 \mathrm{MHz}, \mathrm{CDCl}_{3}, \delta\right): 16.9$ 
(s, $\left.\mathrm{P}(n-\mathrm{Bu})_{3}\right)$; HRMS (ESI-TOF, $m / z$ ): calcd for $\mathrm{C}_{28} \mathrm{H}_{57} \mathrm{O}_{4} \mathrm{P}_{2} \mathrm{Ru}$ : 621.2778; found $621.2828\left[\mathrm{M}-\mathrm{O}_{2} \mathrm{CCH}_{3}\right]^{+}$.

Synthesis of $\mathbf{R u}(\mathrm{CO})_{2}\left(\mathrm{P}(n-\mathrm{Bu})_{3}\right)_{2}\left(\mathrm{O}_{2} \mathrm{CCH}_{2} \mathrm{CH}_{3}\right)_{2}(\mathbf{4 b})$. The title compound was synthesized according to the general procedure by using propionic acid (185 $\mathrm{mg}, 2.50 \mathrm{mmol})$. The raw product was subjected to silica-gel column chromatography using a mixture of $n$-hexane/ethyl acetate (ratio $1: 2, \mathrm{v}: \mathrm{v}$ ) as an eluent to give the respective product as a colorless, crystalline solid. Yield: $366 \mathrm{mg}$ (517 $\mu \mathrm{mol}, 55 \%)$.

M.p. $93{ }^{\circ} \mathrm{C}$; anal. calcd for $\mathrm{C}_{32} \mathrm{H}_{64} \mathrm{O}_{6} \mathrm{P}_{2} \mathrm{Ru}$ : C, 54.30; H, 9.11; found: C, 54.43, H, 9.19; IR data $\left(\mathrm{KBr}, \nu / \mathrm{cm}^{-1}\right): 2962 \mathrm{~m}, 2931 \mathrm{~m}$, 2872 m, 2037 vs, 1969 vs, 1607 s, 1465 m, 1424 m, 1373 m, 1340 m, 1303 w, 1270 m, 1212 w, 1094 m, 1056 w, 905 w, 723 w, 604 w; ${ }^{1} \mathrm{H}-\mathrm{NMR}\left(500 \mathrm{MHz}, \mathrm{CDCl}_{3}, \delta\right): 0.89\left(\mathrm{t},{ }^{3} \mathrm{~J}_{\mathrm{HH}}=7.2 \mathrm{~Hz}, 18 \mathrm{H}\right.$, $\left.\mathrm{CH}_{2} \mathrm{CH}_{3}\right), 1.06\left(\mathrm{t},{ }^{3} \mathrm{~J}_{\mathrm{HH}}=7.6 \mathrm{~Hz}, 6 \mathrm{H}, \mathrm{O}_{2} \mathrm{CCH}_{2} \mathrm{CH}_{3}\right), 1.30-1.48$ (m, 24H, $\mathrm{PCH}_{2} \mathrm{CH}_{2}, \mathrm{CH}_{2} \mathrm{CH}_{3}$ ), 1.73-1.81 (m, 12H, $\mathrm{PCH}_{2}$ ), 2.22 (q, $\left.{ }^{3} J_{\mathrm{HH}}=7.6 \mathrm{~Hz}, 2 \mathrm{H}, \mathrm{O}_{2} \mathrm{CCH}_{2} \mathrm{CH}_{3}\right) ;{ }^{13} \mathrm{C}-\mathrm{NMR}\left(126 \mathrm{MHz}, \mathrm{CDCl}_{3}, \delta\right.$ ): $10.9\left(\mathrm{~s}, \mathrm{O}_{2} \mathrm{CCH}_{2} \mathrm{CH}_{3}\right), 13.7\left(\mathrm{~s}, \mathrm{CH}_{2} \mathrm{CH}_{3}\right), 23.5$ (t, $J_{\mathrm{CP}}=12.8 \mathrm{~Hz}$, $\left.\mathrm{PCH}_{2}\right), 24.5\left(\mathrm{t}, J_{\mathrm{CP}}=6.5 \mathrm{~Hz}, \mathrm{CH}_{2} \mathrm{CH}_{3}\right), 25.1\left(\mathrm{~s}, \mathrm{PCH}_{2} \mathrm{CH}_{2}\right)$, $30.2\left(\mathrm{~s}, \mathrm{O}_{2} \mathrm{CCH}_{2} \mathrm{CH}_{3}\right), 179.8\left(\mathrm{~s}, \mathrm{O}_{2} C\right), 198.0\left(\mathrm{t}, J_{\mathrm{CP}}=11.7 \mathrm{~Hz}\right.$, CO); ${ }^{31} \mathrm{P}-\mathrm{NMR}\left(203 \mathrm{MHz}, \mathrm{CDCl}_{3}, \delta\right): 16.5$ (s, P(n-Bu) $)$ ); HRMS (ESI-TOF, $\mathrm{m} / \mathrm{z}$ ): calcd for $\mathrm{C}_{29} \mathrm{H}_{59} \mathrm{O}_{4} \mathrm{P}_{2} \mathrm{Ru}$ : 635.2935; found 635.2956 $\left[\mathrm{M}-\mathrm{O}_{2} \mathrm{CEt}\right]^{+}$.

Crystal data for $\mathbf{4 b} . \mathrm{C}_{32} \mathrm{H}_{64} \mathrm{O}_{6} \mathrm{P}_{2} \mathrm{Ru}, M_{\mathrm{r}}=707.84$, monoclinic, $P 2 / c, \lambda=0.71073 \AA, a=8.7593(4) \AA, b=13.9460(5) \AA, c=15.6865(5) \AA$, $\beta=95.930(3)^{\circ}, V=1905.97(12) \AA^{3}, Z=2, \rho_{\text {calcd }}=1.233 \mathrm{~g} \mathrm{~cm}^{-3}$, $\mu=0.531 \mathrm{~mm}^{-1}, T=110 \mathrm{~K}, \theta$ range $3.63-26.00^{\circ}, 8850$ reflections collected, 3736 independent reflections $\left(R_{\text {int }}=0.0247\right), R_{1}=$ $0.0318, \mathrm{w} R_{2}=0.0696(I>2 \sigma(I))$.

Synthesis of $\mathrm{Ru}(\mathrm{CO})_{2}\left(\mathrm{P}(n-\mathrm{Bu})_{3}\right)_{2}\left(\mathrm{O}_{2} \mathrm{CCH}\left(\mathrm{CH}_{3}\right)_{2}\right)_{2} \quad$ (4c). The title compound was synthesized according to the general procedure by using isobutyric acid $(220 \mathrm{mg}, 2.50 \mathrm{mmol})$. The raw product was subjected to silica-gel column chromatography using a mixture of $n$-hexane/diethyl ether (ratio $1: 1, \mathrm{v}: \mathrm{v}$ ) as an eluent to give the respective product as a colorless, crystalline solid. Yield: $626 \mathrm{mg}$ (851 $\mu \mathrm{mol}, 91 \%)$.

M.p. $74{ }^{\circ} \mathrm{C}$; anal. calcd (\%) for $\mathrm{C}_{34} \mathrm{H}_{68} \mathrm{O}_{6} \mathrm{P}_{2} \mathrm{Ru}$ : C, 55.49; H, 9.31; found: C, 55.51, H, 9.55; IR data $\left(\mathrm{KBr}, \nu / \mathrm{cm}^{-1}\right): 2958 \mathrm{~m}$, $2931 \mathrm{~m}, 2871 \mathrm{~m}, 2036 \mathrm{vs}, 1969$ vs, $1600 \mathrm{~s}, 1465 \mathrm{~m}, 1322 \mathrm{~m}$, 1386 m, 1360 w, 1339 s, 1302 w, 1271 m, 1209 w, 1092 m, 1055 w, 906 w, 892 w, 802 w, 762 w, 725 w, 604 m; ${ }^{1} \mathrm{H}-\mathrm{NMR}$ $\left(500 \mathrm{MHz} \mathrm{CDCl}_{3}, \delta\right): 0.90\left(\mathrm{t},{ }^{3} J_{\mathrm{HH}}=7.2 \mathrm{~Hz}, 18 \mathrm{H}, \mathrm{CH}_{2} \mathrm{CH}_{3}\right)$, $1.11\left(\mathrm{~d},{ }^{3} J_{\mathrm{HH}}=7.0 \mathrm{~Hz}, 12 \mathrm{H}, \mathrm{CH}\left(\mathrm{CH}_{3}\right)_{2}\right), 1.31-1.50(\mathrm{~m}, 24 \mathrm{H}$, $\left.\mathrm{PCH}_{2} \mathrm{CH}_{2}, \mathrm{CH}_{2} \mathrm{CH}_{3}\right), 1.72-1.84\left(\mathrm{~m}, 12 \mathrm{H}, \mathrm{PCH}_{2}\right), 2.43\left(\mathrm{sep},{ }^{3} J_{\mathrm{HH}}=\right.$ $\left.7.0 \mathrm{~Hz}, 2 \mathrm{H}, \mathrm{CH}\left(\mathrm{CH}_{3}\right)_{2}\right)$; ${ }^{13} \mathrm{C}-\mathrm{NMR}\left(126 \mathrm{MHz} \mathrm{CDCl}_{3}, \delta\right): 13.8$ (s, $\left.\mathrm{CH}_{2} \mathrm{CH}_{3}\right), 20.5\left(\mathrm{~s}, \mathrm{CH}\left(\mathrm{CH}_{3}\right)_{2}\right), 23.5\left(\mathrm{t}, J_{\mathrm{CP}}=12.8 \mathrm{~Hz}, \mathrm{PCH}_{2}\right), 24.5$ $\left(\mathrm{t}, J_{\mathrm{CP}}=6.5 \mathrm{~Hz}, \mathrm{CH}_{2} \mathrm{CH}_{3}\right), 25.3\left(\mathrm{~s}, \mathrm{PCH}_{2} \mathrm{CH}_{2}\right), 36.1\left(\mathrm{~s}, \mathrm{CH}\left(\mathrm{CH}_{3}\right)_{2}\right)$, $182.2\left(\mathrm{~s}, \mathrm{O}_{2} C\right), 198.1$ (t, $\left.J_{\mathrm{CP}}=11.6 \mathrm{~Hz}, C \mathrm{O}\right) ;{ }^{31} \mathrm{P}-\mathrm{NMR}(203 \mathrm{MHz}$, $\mathrm{CDCl}_{3}, \delta$ ): 15.5 (s, $\left.\mathrm{P}(n-\mathrm{Bu})_{3}\right)$; HRMS (ESI-TOF, $\mathrm{m} / \mathrm{z}$ ): calcd for $\mathrm{C}_{34} \mathrm{H}_{68} \mathrm{O}_{6} \mathrm{P}_{2} \mathrm{Ru}+\mathrm{Na}$ : 759.3432; found $759.3380[\mathrm{M}+\mathrm{Na}]^{+}$.

Crystal data for 4 c. $\mathrm{C}_{34} \mathrm{H}_{68} \mathrm{O}_{6} \mathrm{P}_{2} \mathrm{Ru}, M_{\mathrm{r}}=735.89$, monoclinic, $I 2 / a, \lambda=0.71073 \AA, a=22.4889(8) \AA, b=9.0891(4) \AA, c=$ 38.7408(12) $\AA$, $\beta=102.185(3)^{\circ}, V=7740.4(5) \AA^{3}, Z=8, \rho_{\text {calcd }}=$ $1.263 \mathrm{~g} \mathrm{~cm}^{-3}, \mu=0.526 \mathrm{~mm}^{-1}, T=110 \mathrm{~K}, \theta$ range $3.03-25.00^{\circ}$, 20311 reflections collected, 6793 independent reflections $\left(R_{\text {int }}=\right.$ $0.0271), R_{1}=0.0272, \mathrm{w} R_{2}=0.0625(I>2 \sigma(I))$.
Synthesis of $\operatorname{Ru}(\mathrm{CO})_{2}\left(\mathrm{P}(n-\mathrm{Bu})_{3}\right)_{2}\left(\mathrm{O}_{2} \mathrm{CC}\left(\mathrm{CH}_{3}\right)_{3}\right)_{2}$ (4d). The title compound was synthesized according to the general procedure by using pivalic acid $(256 \mathrm{mg}, 2.50 \mathrm{mmol})$. The raw product was subjected to silica-gel column chromatography using a mixture of $n$-hexane/diethyl ether (ratio $2: 1, \mathrm{v}: \mathrm{v}$ ) as an eluent to give the respective product as a colorless, crystalline solid. Yield: $493 \mathrm{mg}$ (645 $\mu \mathrm{mol}, 69 \%)$.

M.p. $48{ }^{\circ} \mathrm{C}$; anal. calcd (\%) for $\mathrm{C}_{36} \mathrm{H}_{72} \mathrm{O}_{6} \mathrm{P}_{2} \mathrm{Ru}$ : C, 56.60; H, 9.50; found: C, 56.83; H, 9.61; IR data ( $\left.\mathrm{KBr}, \nu / \mathrm{cm}^{-1}\right): 2960 \mathrm{~m}, 2930 \mathrm{~m}$, 2872 m, 2864 sh, 2039 vs, 1975 vs, 1606 s, 1565 w, 1478 m, 1466 m, 1461 m, 1420 w, 1390 m, 1333 s, 1213 m, 1093 w, 1048 w, 1007 w, 968 w, 911 w, 888 w, 799 w, 785 w, 727 w, 602 m; ${ }^{1} \mathrm{H}-\mathrm{NMR}$ $\left(500 \mathrm{MHz}, \mathrm{CDCl}_{3}, \delta\right): 0.91\left(\mathrm{t},{ }^{3} J_{\mathrm{HH}}=7.1 \mathrm{~Hz}, 18 \mathrm{H}, \mathrm{CH}_{2} \mathrm{CH}_{3}\right), 1.14$ (s, 18H, $\left.\mathrm{C}\left(\mathrm{CH}_{3}\right)_{3}\right), 1.32-1.49\left(\mathrm{~m}, 24 \mathrm{H}, \mathrm{PCH}_{2} \mathrm{CH}_{2}, \mathrm{CH}_{2} \mathrm{CH}_{3}\right)$, 1.76-1.87 (m, 12H, $\mathrm{PCH}_{2}$ ); ${ }^{13} \mathrm{C}-\mathrm{NMR}\left(126 \mathrm{MHz} \mathrm{CDCl}_{3}, \delta\right): 13.8$ $\left(\mathrm{s}, \mathrm{CH}_{2} \mathrm{CH}_{3}\right), 23.3\left(\mathrm{t}, J_{\mathrm{CP}}=12.7 \mathrm{~Hz}, \mathrm{PCH}_{2}\right), 24.5\left(\mathrm{t}, J_{\mathrm{CP}}=6.4 \mathrm{~Hz}\right.$, $\left.\mathrm{CH}_{2} \mathrm{CH}_{3}\right), 25.4\left(\mathrm{~s}, \mathrm{PCH}_{2} \mathrm{CH}_{2}\right), 28.9\left(\mathrm{~s}, \mathrm{C}\left(\mathrm{CH}_{3}\right)_{3}\right), 39.5\left(\mathrm{~s}, \mathrm{C}\left(\mathrm{CH}_{3}\right)_{3}\right)$, $183.5\left(\mathrm{~s}, \mathrm{O}_{2} C\right), 198.2\left(\mathrm{t}, J_{\mathrm{CP}}=11.7 \mathrm{~Hz}, C \mathrm{O}\right) ;{ }^{31} \mathrm{P}-\mathrm{NMR}(203 \mathrm{MHz}$, $\left.\mathrm{CDCl}_{3}, \delta\right): 14.7$ (s, $\left.\mathrm{P}(n \text {-Bu })_{3}\right)$; HRMS (ESI-TOF, $\left.m / z\right)$ : calcd for $\mathrm{C}_{31} \mathrm{H}_{63} \mathrm{O}_{4} \mathrm{P}_{2} \mathrm{Ru}$ : 663.3248; found 663.3376 [M-O $\left.\mathrm{O}_{2} \mathrm{C}^{t} \mathrm{Bu}\right]^{+}$.

Synthesis of $\mathrm{Ru}(\mathrm{CO})_{2}\left(\mathrm{P}(n-\mathrm{Bu})_{3}\right)_{2}\left(\mathrm{O}_{2} \mathrm{CCH}_{2} \mathrm{OCH}_{3}\right)_{2}$ (4e). The title compound was synthesized according to the general procedure by using 2-methoxyacetic acid (225 $\mathrm{mg}, 2.50 \mathrm{mmol}$ ). The raw product was subjected to silica-gel column chromatography using a mixture of $n$-hexane/ethyl acetate (ratio $1: 2, \mathrm{v}: \mathrm{v}$ ) as an eluent to give the respective product as a colorless, crystalline solid. Yield: $578 \mathrm{mg}(781 \mu \mathrm{mol}, 83 \%)$.

M.p. $96{ }^{\circ} \mathrm{C}$; anal. calcd (\%) for $\mathrm{C}_{32} \mathrm{H}_{64} \mathrm{O}_{8} \mathrm{P}_{2} \mathrm{Ru}$ : C, 51.95; H, 8.72; found: C, 52.13; H, 9.14; IR data $\left(\mathrm{KBr}, \nu / \mathrm{cm}^{-1}\right): 2959 \mathrm{~m}$, 2930 m, 2871 m, 2815 w, 2037 vs, 1969 vs, 1645 s, 1615 m, 1467 w, 1422 w, 1397 w, 1376 m, 1334 w, 1293 m, 1277 w, 1213 w, 1196 w, 1128 s, 1093 w, 1055 w, 948 w, 904 w, 797 w, 776 w, 723 w, $605 \mathrm{w}, 594 \mathrm{w} ;{ }^{1} \mathrm{H}-\mathrm{NMR}\left(500 \mathrm{MHz}, \mathrm{CDCl}_{3}, \delta\right): 0.92\left(\mathrm{t},{ }^{3} J_{\mathrm{HH}}=7.1 \mathrm{~Hz}\right.$, $\left.18 \mathrm{H}, \mathrm{CH}_{2} \mathrm{CH}_{3}\right), 1.33-1.49\left(\mathrm{~m}, 24 \mathrm{H}, \mathrm{PCH}_{2} \mathrm{CH}_{2}, \mathrm{CH}_{2} \mathrm{CH}_{3}\right), 1.79-1.87$ $\left(\mathrm{m}, 12 \mathrm{H}, \mathrm{PCH}_{2}\right), 3.41\left(\mathrm{~s}, 6 \mathrm{H}, \mathrm{OCH}_{3}\right), 3.89\left(\mathrm{~s}, 4 \mathrm{H}, \mathrm{CH}_{2} \mathrm{O}\right) ;{ }^{13} \mathrm{C}-\mathrm{NMR}$ $\left(126 \mathrm{MHz}, \mathrm{CDCl}_{3}, \delta\right): 13.8\left(\mathrm{~s}, \mathrm{CH}_{2} \mathrm{CH}_{3}\right), 23.6\left(\mathrm{t}, J_{\mathrm{CP}}=13.0 \mathrm{~Hz}\right.$, $\left.\mathrm{PCH}_{2}\right), 24.5\left(\mathrm{t}, J_{\mathrm{CP}}=6.6 \mathrm{~Hz}, \mathrm{CH}_{2} \mathrm{CH}_{3}\right), 25.2\left(\mathrm{~s}, \mathrm{PCH}_{2} \mathrm{CH}_{2}\right), 59.0$ $\left(\mathrm{s}, \mathrm{OCH}_{3}\right), 72.1\left(\mathrm{~s}, \mathrm{CH}_{2} \mathrm{O}\right), 175.5\left(\mathrm{~s}, \mathrm{O}_{2} C\right), 197.5\left(\mathrm{t}, J_{\mathrm{CP}}=11.6 \mathrm{~Hz}\right.$, CO); ${ }^{31} \mathrm{P}-\mathrm{NMR}\left(203 \mathrm{MHz}, \mathrm{CDCl}_{3}, \delta\right.$ ): 16.7 (s); HRMS (ESI-TOF, $m / z$ ): calcd for $\mathrm{C}_{32} \mathrm{H}_{64} \mathrm{O}_{8} \mathrm{P}_{2} \mathrm{Ru}+\mathrm{K}$ : 779.2760; found 779.2831 $[\mathrm{M}+\mathrm{K}]^{+}$.

Crystal data for 4e. $\mathrm{C}_{32} \mathrm{H}_{64} \mathrm{O}_{8} \mathrm{P}_{2} \mathrm{Ru}, M_{\mathrm{r}}=739.84$, monoclinic, $C 2 / c, \lambda=1.54184 \AA$, $a=8.3401(8) \AA \circ, b=31.639(3) \AA, c=15.0063(16) \AA$, $\beta=91.517(11)^{\circ}, V=3958.4(7) \AA^{3}, Z=4, \rho_{\text {calcd }}=1.241 \mathrm{~g} \mathrm{~cm}^{-3}$, $\mu=4.306 \mathrm{~mm}^{-1}, T=110 \mathrm{~K}, \theta$ range $4.06-67.22^{\circ}, 10925$ reflections collected, 3485 independent reflections $\left(R_{\text {int }}=0.0431\right), R_{1}=0.0488$, $\mathrm{w} R_{2}=0.1303(I>2 \sigma(I))$.

Synthesis of $\mathbf{R u}(\mathrm{CO})_{2}\left(\mathrm{P}(\boldsymbol{n}-\mathrm{Bu})_{3}\right)_{2}\left(\mathrm{O}_{2} \mathbf{C C F}_{3}\right)_{2}(\mathbf{4 f})$. The title compound was synthesized according to the general procedure by using trifluoroacetic acid ( $285 \mathrm{mg}, 2.50 \mathrm{mmol})$. The raw product was subjected to silica-gel column chromatography using a mixture of $n$-hexane/diethyl ether (ratio $9: 1, \mathrm{v}: \mathrm{v}$ ) as an eluent to give the respective product as a colorless, crystalline solid. Yield: $441 \mathrm{mg}$ (560 $\mu \mathrm{mol}, 60 \%)$.

M.p. $100{ }^{\circ} \mathrm{C}$; anal. calcd (\%) for $\mathrm{C}_{30} \mathrm{H}_{54} \mathrm{~F}_{6} \mathrm{O}_{6} \mathrm{P}_{2} \mathrm{Ru}$ : C, 45.74; $\mathrm{H}$, 6.91; found: C, 45.76; H, 6.94; IR data $\left(\mathrm{KBr}, \nu / \mathrm{cm}^{-1}\right): 2966 \mathrm{~m}$, 
$2934 \mathrm{~m}, 2878 \mathrm{~m}, 2052$ vs, 1988 vs, 1688 s, $1467 \mathrm{~m}, 1411 \mathrm{~m}$, 1384 w, 1194 s, 1145 s, 1090 w, 1055 w, 967 w, 906 w, 848 m, 806 w, $792 \mathrm{~m}, 775 \mathrm{w}, 728 \mathrm{~m}, 600 \mathrm{~m} ;{ }^{1} \mathrm{H}-\mathrm{NMR}\left(500 \mathrm{MHz}, \mathrm{CDCl}_{3}, \delta\right): 0.92$ $\left(\mathrm{t},{ }^{3} J_{\mathrm{HH}}=7.1 \mathrm{~Hz}, 18 \mathrm{H}, \mathrm{CH}_{2} \mathrm{CH}_{3}\right), 1.33-1.49\left(\mathrm{~m}, 24 \mathrm{H}, \mathrm{PCH}_{2} \mathrm{CH}_{2}\right.$, $\left.\mathrm{CH}_{2} \mathrm{CH}_{3}\right), 1.77-1.87\left(\mathrm{~m}, 12 \mathrm{H}, \mathrm{PCH}_{2}\right) ;{ }^{13} \mathrm{C}-\mathrm{NMR}(126 \mathrm{MHz}$, $\mathrm{CDCl}_{3}, \delta$ ): $13.6\left(\mathrm{~s}, \mathrm{CH}_{2} \mathrm{CH}_{3}\right), 23.5\left(\mathrm{t}, J_{\mathrm{CP}}=13.2 \mathrm{~Hz}, \mathrm{PCH}_{2}\right), 24.3$ $\left(\mathrm{t}, J_{\mathrm{CP}}=6.8 \mathrm{~Hz}, \mathrm{CH}_{2} \mathrm{CH}_{3}\right), 25.0\left(\mathrm{~s}, \mathrm{PCH}_{2} \mathrm{CH}_{2}\right), 115.6\left(\mathrm{q},{ }^{1} J_{\mathrm{CF}}=\right.$ $\left.290.1 \mathrm{~Hz}, C \mathrm{~F}_{3}\right), 162.7\left(\mathrm{q},{ }^{2} J_{\mathrm{CF}}=36.9 \mathrm{~Hz}, \mathrm{O}_{2} C\right), 196.4\left(\mathrm{t}, J_{\mathrm{CP}}=\right.$ $11.2 \mathrm{~Hz}, C \mathrm{O}) ;{ }^{31} \mathrm{P}-\mathrm{NMR}\left(203 \mathrm{MHz}, \mathrm{CDCl}_{3}, \delta\right): 15.9\left(\mathrm{~s}, \mathrm{P}(n-\mathrm{Bu})_{3}\right)$; HRMS (ESI-TOF, $m / z$ ): calcd for $\mathrm{C}_{30} \mathrm{H}_{54} \mathrm{~F}_{6} \mathrm{O}_{6} \mathrm{P}_{2} \mathrm{Ru}+\mathrm{Na}$ : 811.2244; found $811.2187[\mathrm{M}+\mathrm{Na}]^{+}$.

Crystal data for $4 f$. $\mathrm{C}_{30} \mathrm{H}_{54} \mathrm{~F}_{6} \mathrm{O}_{6} \mathrm{P}_{2} \mathrm{Ru}, M_{\mathrm{r}}=787.74$, monoclinic, $P 2_{1} / c, \lambda=0.71073 \AA, a=18.0972(4) \AA, \quad b=14.0559(3) \AA$, $c=15.2263(3) \AA ̊=94.708(2)^{\circ}, V=3860.08(14) \AA^{3}, Z=4, \rho_{\text {calcd }}=$ $1.355 \mathrm{~g} \mathrm{~cm}^{-3}, \mu=0.554 \mathrm{~mm}^{-1}, T=110 \mathrm{~K}, \theta$ range $2.90-25.00^{\circ}$, 19426 reflections collected, 6781 independent reflections $\left(R_{\mathrm{int}}=\right.$ $0.0271), R_{1}=0.0366, \mathrm{w} R_{2}=0.0779(I>2 \sigma(I))$.

Synthesis of $\operatorname{Ru}(\mathbf{C O})_{2}\left(\mathbf{P}(n-B u)_{3}\right)_{2}\left(\mathbf{O}_{2} C_{C_{2}} F_{5}\right)_{2}$ (4g). The title compound was synthesized according to the general procedure by using pentafluoropropionic acid (411 $\mathrm{mg}, 2.50 \mathrm{mmol}$ ). The raw product was subjected to silica-gel column chromatography using a mixture of $n$-hexane/diethyl ether (ratio 9:1, $\mathrm{v}: \mathrm{v}$ ) as an eluent to give the respective product as a colorless, crystalline solid. Yield: $713 \mathrm{mg}(802 \mu \mathrm{mol}, 86 \%)$.

M.p. $93{ }^{\circ} \mathrm{C}$; anal. calcd (\%) for $\mathrm{C}_{32} \mathrm{H}_{54} \mathrm{~F}_{10} \mathrm{O}_{6} \mathrm{P}_{2} \mathrm{Ru}$ : C, 43.29; $\mathrm{H}, 6.13$; found: $\mathrm{C}, 43.26, \mathrm{H}, 6.23$; IR data $\left(\mathrm{KBr}, \nu / \mathrm{cm}^{-1}\right): 2962 \mathrm{~m}$, 2937 m, 2876 m, 2052 vs, 1987 vs, 1681 s, 1469 m, 1425 m, 1383 m, 1337 m, 1219 s, 1170 s, 1145 s, 1093 m, 1053 w, 1030 m, 906 w, $821 \mathrm{~m}, 801 \mathrm{w}, 776 \mathrm{w}, 736 \mathrm{~m}, 721 \mathrm{~m}, 599 \mathrm{~m} ;{ }^{1} \mathrm{H}-\mathrm{NMR}(500 \mathrm{MHz}$, $\left.\mathrm{CDCl}_{3}, \delta\right): 0.91\left(\mathrm{t},{ }^{3} J_{\mathrm{HH}}=7.0 \mathrm{~Hz}, 18 \mathrm{H}, \mathrm{CH}_{2} \mathrm{CH}_{3}\right), 1.33-1.47(\mathrm{~m}, 24 \mathrm{H}$, $\left.\mathrm{PCH}_{2} \mathrm{CH}_{2}, \mathrm{CH}_{2} \mathrm{CH}_{3}\right), 1.78-1.87\left(\mathrm{~m}, 12 \mathrm{H}, \mathrm{PCH}_{2}\right) ;{ }^{13} \mathrm{C}-\mathrm{NMR}$ $\left(126 \mathrm{MHz}_{\mathrm{CDCl}}, \delta\right): 13.6\left(\mathrm{~s}, \mathrm{CH}_{2} \mathrm{CH}_{3}\right), 23.4\left(\mathrm{t}, J_{\mathrm{CP}}=13.2 \mathrm{~Hz}\right.$, $\mathrm{PCH}_{2}$ ), 24.3 (t, $\left.J_{\mathrm{CP}}=6.7 \mathrm{~Hz}, \mathrm{CH}_{2} \mathrm{CH}_{3}\right), 25.0\left(\mathrm{~s}, \mathrm{PCH}_{2} \mathrm{CH}_{2}\right), 106.3$ $\left(\mathrm{tq},{ }^{1} J_{\mathrm{CF}}=264.0 \mathrm{~Hz},{ }^{2} J_{\mathrm{CF}}=37.9 \mathrm{~Hz}, C \mathrm{~F}_{2}\right), 118.7\left(\mathrm{qt},{ }^{1} J_{\mathrm{CF}}=\right.$ $\left.285.5 \mathrm{~Hz},{ }^{2} J_{\mathrm{CF}}=35.1 \mathrm{~Hz}, C \mathrm{~F}_{3}\right), 163.1\left(\mathrm{t},{ }^{2} J_{\mathrm{CF}}=26.0 \mathrm{~Hz}, \mathrm{O}_{2} C\right)$, $196.5\left(\mathrm{t}, J_{\mathrm{CP}}=11.2 \mathrm{~Hz}, C \mathrm{O}\right) ;{ }^{31} \mathrm{P}-\mathrm{NMR}\left(203 \mathrm{MHz}, \mathrm{CDCl}_{3}, \delta\right): 15.1$ (s, $\left.\mathrm{P}(n-\mathrm{Bu})_{3}\right)$; HRMS (ESI-TOF, $\left.m / z\right)$ : calcd for $\mathrm{C}_{32} \mathrm{H}_{54} \mathrm{~F}_{10} \mathrm{O}_{6} \mathrm{P}_{2} \mathrm{Ru}+\mathrm{Na}$ : 911.2181; found 911.2090 [M + Na $]^{+}$.

Crystal data for $\mathbf{4 g} . \mathrm{C}_{32} \mathrm{H}_{54} \mathrm{~F}_{10} \mathrm{O}_{6} \mathrm{P}_{2} \mathrm{Ru}, M_{\mathrm{r}}=887.76$, monoclinic, $I 2 / a, \lambda=0.71073 \AA, a=23.1780(5) \AA, b=9.2295(3) \AA, c=38.6832(8) \AA$, $\beta=102.737(2)^{\circ}, V=8071.5(4) \AA^{3}, Z=8, \rho_{\text {calcd }}=1.461 \mathrm{~g} \mathrm{~cm}^{-3}$, $\mu=0.552 \mathrm{~mm}^{-1}, T=110 \mathrm{~K}, \theta$ range $2.97-25.00^{\circ}, 35399$ reflections collected, 7072 independent reflections $\left(R_{\mathrm{int}}=0.0365\right), R_{1}=0.0307$, $\mathrm{w} R_{2}=0.0780(I>2 \sigma(I))$.

\section{Results and discussion}

\section{Synthesis and characterization}

Ruthenium complexes $\mathrm{Ru}(\mathrm{CO})_{2}\left(\mathrm{P}(n-\mathrm{Bu})_{3}\right)_{2}\left(\mathrm{O}_{2} \mathrm{CR}\right)_{2}(4 \mathrm{a}, \mathrm{R}=\mathrm{Me}$; 4b, $\mathrm{R}=\mathrm{Et} ; \mathbf{4 c}, \mathrm{R}=\mathrm{i}-\mathrm{Pr} ; \mathbf{4 d}, \mathrm{R}=t$-Bu; 4e, $\mathrm{R}=\mathrm{CH}_{2} \mathrm{OCH}_{3} ; \mathbf{4 f}$, $\mathrm{R}=\mathrm{CF}_{3} ; \mathbf{4 g}, \mathrm{R}=\mathrm{CF}_{2} \mathrm{CF}_{3}$ ) were prepared by reacting $\mathrm{Ru}_{3}(\mathrm{CO})_{12}(\mathbf{1})$ with $\mathrm{P}(n-\mathrm{Bu})_{3}(2)$ and the respective carboxylic acid (3) analogously to a synthetic methodology described by Bianchi ${ }^{25}$ (Scheme 1). After an appropriate work-up, organometallic compounds $\mathbf{4 a - g}$ could be isolated in good to excellent yields as air and moisture stable colorless solids, which dissolve in most of the common

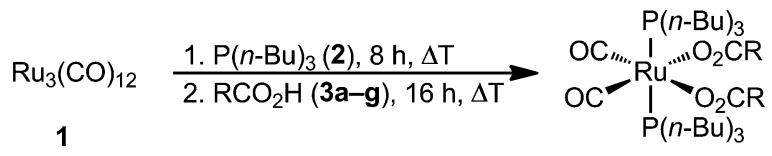

4a-g

a: $\mathrm{R}=\mathrm{Me} ; \mathbf{b}: \mathrm{R}=\mathrm{Et} ; \mathbf{c}: i-\mathrm{Pr} ; \mathbf{d}: \mathrm{R}=t-\mathrm{Bu} ; \mathbf{e}: \mathrm{R}=\mathrm{CH}_{2} \mathrm{OCH}_{3}$;

f: $\mathrm{R}=\mathrm{CF}_{3} ; \mathbf{g}: \mathrm{R}=\mathrm{CF}_{2} \mathrm{CF}_{3}$

Scheme 1 Synthesis of ruthenium complexes $\mathbf{4 a - g}$

organic solvents including methanol, dichloromethane, toluene, diethyl ether and $n$-hexane.

The identities of all compounds were confirmed by elemental analysis, IR and NMR $\left({ }^{1} \mathrm{H},{ }^{13} \mathrm{C}\left\{{ }^{1} \mathrm{H}\right\},{ }^{31} \mathrm{P}\left\{{ }^{1} \mathrm{H}\right\}\right)$ spectroscopy and high resolution ESI mass spectrometry (Experimental section). The molecular structures of $\mathbf{4 b}, \mathbf{4 c}$ and $\mathbf{4 e - g}$ in the solid state were determined by single crystal X-ray structure analysis. In addition, the thermal behavior was studied by vapor pressure and thermogravimetric measurements.

The ${ }^{31} \mathrm{P}\left\{{ }^{1} \mathrm{H}\right\}$ NMR spectra of ruthenium complexes $4 \mathbf{4}-\mathbf{g}$ exhibit one characteristic singlet for the $\mathrm{P}(n-\mathrm{Bu})_{3}$ groups ranging from 14.7 to $16.7 \mathrm{ppm}$ (Experimental section). As the respective resonance signals are shifted to higher fields as compared to $2(-30.9 \mathrm{ppm}),{ }^{31} \mathrm{P}\left\{{ }^{1} \mathrm{H}\right\}$ NMR spectroscopy is suitable to monitor the progress of the reactions. The ${ }^{1} \mathrm{H}$ and ${ }^{13} \mathrm{C}\left\{{ }^{1} \mathrm{H}\right\}$ NMR spectra of $\mathbf{4 a}-\mathbf{g}$ are in accordance with the proposed structures. Unique to the ${ }^{13} \mathrm{C}\left\{{ }^{1} \mathrm{H}\right\}$ NMR spectra is the splitting of the $\alpha$ - and $\beta-\mathrm{CH}_{2}$ groups of the $\mathrm{P}(n-\mathrm{Bu})_{3}$ ligand into triplets (Experimental section), which is a common phenomenon for complexes containing trans-phosphine ligands. ${ }^{26,27}$ This finding is confirmed by the calculations of Metzinger ${ }^{28}$ and Harris. ${ }^{29}$

In the IR spectra of ruthenium complexes $\mathbf{4 a}-\mathbf{g}$, two strong stretching vibrations for the terminal carbonyl groups are observed between 1969 and $2057 \mathrm{~cm}^{-1}$ (Experimental section). The number of CO vibrations reveals that the carbonyl groups have to adopt a cis-arrangement in the octahedral coordination sphere of the $\mathrm{Ru}(\mathrm{II})$ ion, as for a trans isomer just one strong carbonyl stretching is expected. ${ }^{30}$ From the difference of the characteristic asymmetric $\left(\tilde{v}_{\text {asym }}\right)$ and symmetric $\left(\tilde{v}_{\text {sym }}\right)$ carboxylate stretching vibrations $\left(\Delta \tilde{v}, \Delta \tilde{v}=\tilde{v}_{\text {asym }}-\tilde{v}_{\text {asym }}\right)$ one can estimate the structural bonding motif of the carboxylate ligands. ${ }^{31}$ For ruthenium complexes $4 \mathbf{a}-\mathbf{g}$ large $\Delta \tilde{v}$ values of over $250 \mathrm{~cm}^{-1}$ indicate a monodentate coordination of the carboxylate groups to the $\mathrm{Ru}(\mathrm{II})$ ion, which was confirmed by single X-ray structure determination (see below).

The structures of $\mathbf{4 b}, \mathbf{c}$ and $\mathbf{4} \mathbf{e}-\mathbf{g}$ in the solid state were determined by single crystal X-ray diffraction analysis. Suitable crystals were obtained from a concentrated $n$-hexane solution at $5{ }^{\circ} \mathrm{C}$. The ORTEP diagrams of complexes $\mathbf{4 b}, \mathbf{c}, \mathbf{e}$ are presented in Fig. 1. The molecular structures of complexes $\mathbf{4 f}, \mathbf{g}$ along with key structural data can be found in the ESI. $\dagger$ The crystal and structure refinement data are presented in the Experimental section.

All compounds crystallize in monoclinic space groups (4b: $P 2 / c$; 4c,h: $I 2 / a$; 4e: $C 2 / c$; 4 f: $P 2_{1} / c$ ) with one molecule in the asymmetric unit, except for $\mathbf{4 b}$ and $\mathbf{4 e}$ with one half of the compound and a 

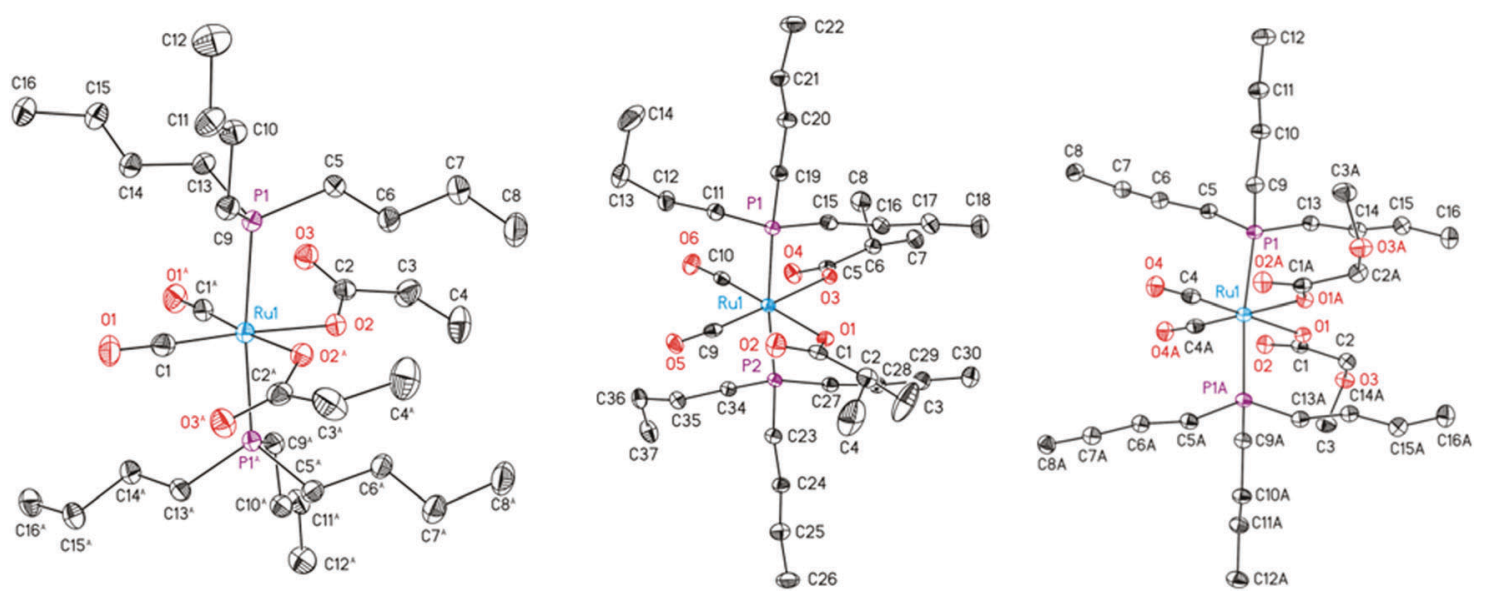

Fig. 1 ORTEP diagrams (30\% probability level) of the molecular structures of $\mathbf{4 b}$ (left), $\mathbf{4 c}$ (middle) and $\mathbf{4 e}$ (right, 50\% probability level) with the atom numbering scheme. All hydrogen and disordered atoms have been omitted for clarity (symmetry code for $4 \mathbf{b}: A=-x+1, y,-z+3 / 2 ;$ for $4 \mathbf{e}: A=-x+2, y$, $-z+1 / 2)$.

$C_{2}$-symmetry axis through the Ru atom. The complexes consist of a slightly distorted octahedral coordinated ruthenium atom with the two trans-positioned tri- $n$-butylphosphines (P1 and P2) in the apical positions, two cis-oriented carbonyls and two cis-monodentate O-bonded carboxylates in the equatorial plane (Fig. 1).

The tri- $n$-butyl coordinated complexes $4 \mathbf{b}$ and $4 c$ and $4 \mathbf{e}-\mathbf{g}$ exhibit a similar structural behavior, compared to the recently reported triphenyl derivatives, ${ }^{26,27}$ e.g. a slight bending of the phosphines towards the carboxylate moieties ( $\mathrm{P}-\mathrm{Ru}-\mathrm{P}$ angles between 171.11(2) in $\mathbf{4 g}$ and $176.23(3)^{\circ}$ in $\left.\mathbf{4 f}\right)$. The $\mathrm{CO}_{2}$-planes are rotated out of the central coordination plane by 24.1(2) up to $32.9(3)^{\circ}$ except for the more coplanar $4 \mathbf{e}\left(10.6(3)^{\circ}\right)$ and all carbonyl oxygens are directed towards the carbonyl substituents. The angles between the carboxylates and carbonyls differ between each derivative (O-Ru-O: 79.27(11) in 4 e to $83.75(6)^{\circ}$ in $\mathbf{4 g}$; C-Ru-C: $87.13(19)$ in $4 \mathbf{e}$ to $89.94(13)^{\circ}$ in $\left.\mathbf{4 f}\right)$. However, a trend based on electronic or steric effects is not present. The Ru-P bond distances of electronically poor complexes $\mathbf{4 f}$ and $\mathbf{4 g}$ are increased to at least 2.3993(6) A compared to electron-rich $\mathbf{4 b}, \mathbf{c}, \mathbf{e}$ in which they do not exceed 2.3910(5) $\AA$. The $n$-butyl moieties of both phosphine substituents reveal a staggered rotation between $171.47(15)(\mathbf{4 f})$ and $179.53(15)^{\circ}(\mathbf{4 b})$.

\section{Thermal behavior and vapor pressure measurements}

To gain first information on the thermal behavior of the designed precursor complexes, compounds $\mathbf{4 a - g}$ were studied by thermogravimetric (TG) analysis. The physical properties of 4a-g are summarized in Table 1 . The melting points of the complexes could be tuned by changing the carboxylate ligands. In this respect, the melting points decrease with the increasing chain length or branching of the carboxylate ligand from $108^{\circ} \mathrm{C}$ in $4 \mathrm{a}$ to $48{ }^{\circ} \mathrm{C}$ in $4 \mathrm{~d}$. TG measurements were carried out in a

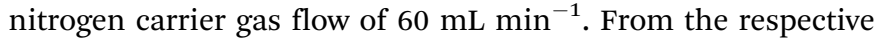
TG traces it can be seen that all compounds show a weight loss between 220 and $350{ }^{\circ} \mathrm{C}$ which results from an overlapped process of precursor evaporation and decomposition (Fig. 2).
Table 1 Physical properties of Ru precursors $\mathbf{4 a - g}$

\begin{tabular}{|c|c|c|c|c|c|c|}
\hline \multirow[b]{2}{*}{ Precursor } & \multirow{2}{*}{$\begin{array}{l}\text { M.p. } \\
{\left[{ }^{\circ} \mathrm{C}\right]}\end{array}$} & \multirow{2}{*}{$\begin{array}{l}\text { Onset } \\
\text { temp. }\left[{ }^{\circ} \mathrm{C}\right]\end{array}$} & \multicolumn{3}{|c|}{$\underline{\log p[\mathrm{bar}]=A-B / T^{a}}$} & \multirow{2}{*}{$\begin{array}{l}\Delta H_{\mathrm{vap}}(\exp .) \\
{\left[\mathrm{kJ} \mathrm{mol}^{-1}\right]}\end{array}$} \\
\hline & & & $A$ & $B$ & $R^{2}$ & \\
\hline $4 a$ & 108 & 247 & 7.93 & 4918 & 0.9986 & 122.4 \\
\hline $4 b$ & 93 & 244 & 8.64 & 5216 & 0.9962 & 107.8 \\
\hline $4 c$ & 74 & 254 & 9.04 & 5330 & 0.9782 & 119.1 \\
\hline 4d & 48 & 268 & 7.42 & 4602 & 0.9868 & 123.7 \\
\hline $4 e$ & 96 & 258 & 9.52 & 5349 & 0.9768 & 95.9 \\
\hline $4 \mathrm{f}$ & 100 & 277 & 9.19 & 5331 & 0.9777 & 100.3 \\
\hline $4 g$ & 93 & 275 & 8.54 & 5135 & 0.9828 & 111.1 \\
\hline
\end{tabular}

${ }^{a} A$ and $B=$ Antoine parameters; $T=$ absolute temperature; $R^{2}=$ coefficient of determination.

Thereby, the appearance of the TG traces is strongly dependent on the applied heating rate (ESI $\dagger$ ). A heating rate of $10 \mathrm{~K} \mathrm{~min}^{-1}$ was employed to ensure that the precursor does not evaporate completely and hence information on the decomposition temperature of the precursor can be received. A lower heating rate leads to the evaporation of the preferred compounds, which is beneficial for the vapor pressure measurements (see below). ${ }^{32,33}$ The TG traces also indicate that marginal changes in the periphery of the complex, for example, the modification of the carboxylate ligands, do not have a significant effect on the evaporation and decomposition process. This observation is also confirmed by the similarity of the respective onset temperatures ranging from $244{ }^{\circ} \mathrm{C}$ for $\mathbf{4 b}$ to $277{ }^{\circ} \mathrm{C}$ for $\mathbf{4 f}$ (Table 1 ).

Vapor pressure measurements of ruthenium complexes $\mathbf{4 a -} \mathbf{g}$ were carried out over a temperature range from 100 to $240{ }^{\circ} \mathrm{C}$ under atmospheric pressure using a TGA system with a horizontal balance. The details of the applied method have been published previously. ${ }^{2}$ All vapor pressure measurements were performed thrice to provide a statistic validation of the experimental data. From the respective vapor pressure traces it can be seen that the different carboxylate ligands influence the vapor pressure of the resulting precursor complex (Fig. 3). The highest volatility was observed for compound $4 \mathbf{e}$, featuring a $\mathrm{CH}_{2} \mathrm{OCH}_{3}$ substituent. As expected, the $\mathrm{CF}_{3}$ groups in $\mathbf{4 f}$ also increase the volatility due 

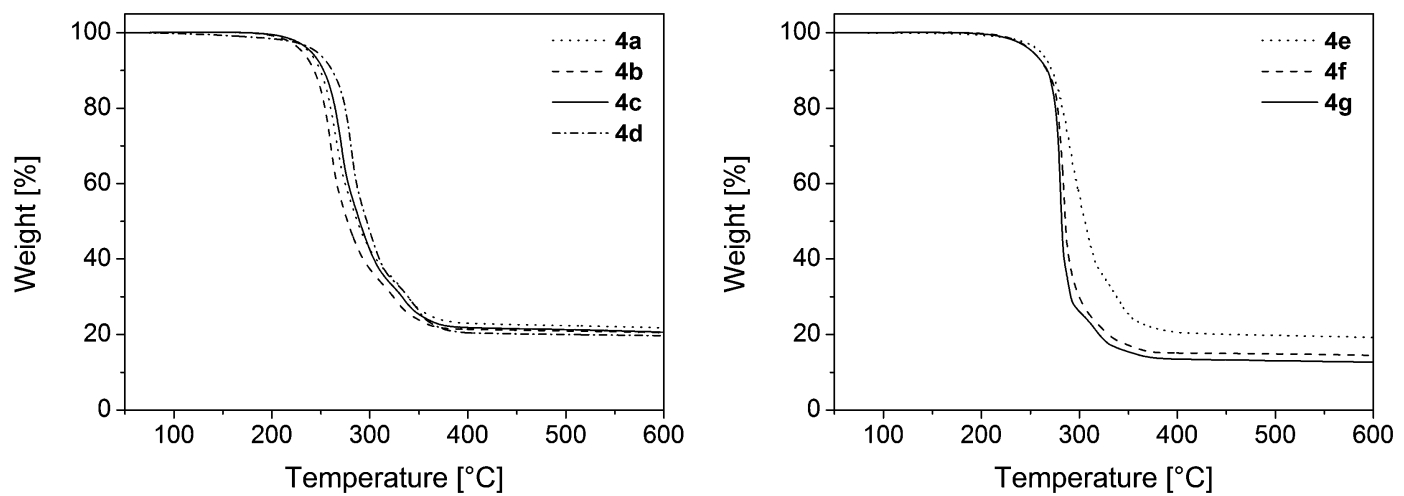

Fig. 2 TG traces of $\mathbf{4 a - d}$ (left) and $\mathbf{4 e - g}$ (right); gas flow, $\mathrm{N}_{2} 60 \mathrm{~mL} \mathrm{~min}^{-1}$, heating rate, $10 \mathrm{~K} \mathrm{~min}^{-1}$.

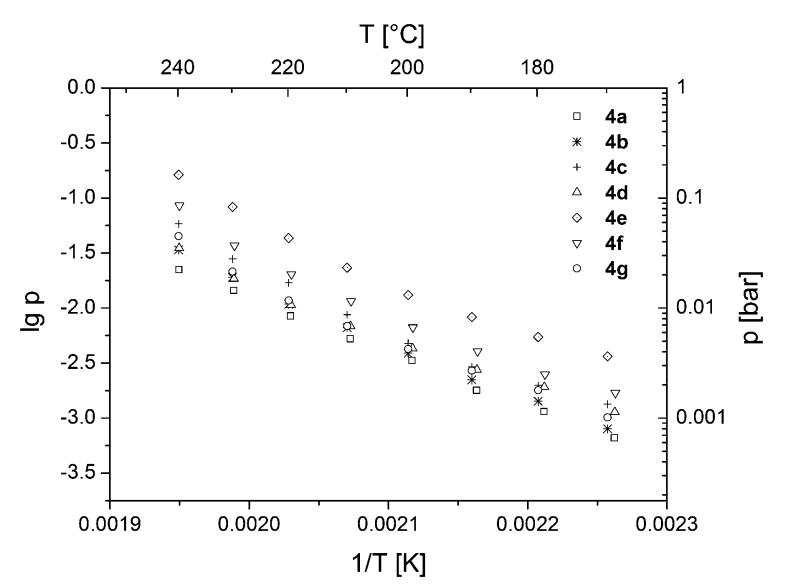

Fig. 3 Vapor pressure traces of ruthenium complexes $\mathbf{4 a - g}$

to the low polarizability of the $\mathrm{C}-\mathrm{F}$ bonds, which leads to reduced intermolecular interactions between the complexes. ${ }^{34}$ However, the prolonged $\mathrm{CF}_{2} \mathrm{CF}_{3}$ substitution in $\mathbf{4 g}$ resulted in a decrease of the volatility. The vapor pressures of the remaining compounds $\mathbf{4 a - d}$ hardly differ from each other. In comparison to the previously reported vapor pressures of substituted ruthenocenes and half-open ruthenocenes, ${ }^{2}$ the reported ruthenium complexes 4a-g possess quite similar volatilities.

\section{Chemical vapor deposition experiments}

Ruthenium complexes $\mathbf{4 a - f}$ have been successfully applied in CVD experiments for the deposition of phosphorus-doped ruthenium layers. The experiments were carried out in a home-built vertical cold-wall reactor equipped with a continuous evaporation system. Deposition was performed using nitrogen as a carrier gas (30-

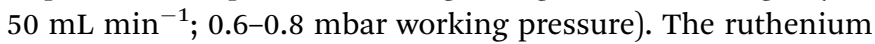
films were deposited on Si wafers, which were covered by a continuous $100 \mathrm{~nm}$ thick thermal $\mathrm{SiO}_{2}$ layer. Additionally, precursor 4e was deposited on a trench patterned $\mathrm{SiO}_{2}$ wafer in order to study the step coverage properties.

For appropriate evaporation of the precursor complexes, temperatures of $120-135{ }^{\circ} \mathrm{C}$ in the vaporizer unit were needed. Furthermore, the glass lines were heated to approximately $100{ }^{\circ} \mathrm{C}$. The substrate temperatures were selected according to the results of the TG analyses. Decomposition of the precursor complexes starts at around $270{ }^{\circ} \mathrm{C}$, but a minimum substrate temperature of $350{ }^{\circ} \mathrm{C}$ is required in all cases for complete precursor deposition and formation of ruthenium layers. Therefore, deposition was carried out using substrate temperatures between 350 and $400{ }^{\circ} \mathrm{C}$. The MOCVD deposition parameters of the obtained layers A-H are summarized in Table 2. The deposition was monitored visually and terminated after the formation of metallic layers. Depending on the experimental conditions, 25-65 nm thick uniform layers were obtained. The $40-55 \mathrm{~nm}$ thin films are reflective metallic layers, whereas thinner films possess a slightly bluish appearance and thicker films show a yellowish tinge.

\section{Layer characterization}

The scanning electron microscopy (SEM) images of the films deposited from $\mathbf{4 a - f}$ evidence the formation of dense and conformal layers for all precursors (Fig. 4). In general, the morphology of the produced layers A-F is very similar, so that no optical differences in surface roughness or homogeneity could be observed. The film thicknesses between 25 and $65 \mathrm{~nm}$ were determined by cross-sectional SEM images (ESI $\dagger$ ). Thereby, the highest growth rates were obtained for $\mathbf{4 e}$ and $\mathbf{4 f}$, possessing the highest volatilities according to the vapor pressure measurements (Fig. 3). The deposition rate can be enhanced by higher deposition temperatures and carrier gas flow rates (layers $\mathrm{E}$ and $\mathrm{F}$, Table 2).

Table 2 Deposition parameters of Ru layers A-H deposited from $\mathbf{4 a - f}$

\begin{tabular}{|c|c|c|c|c|c|c|c|}
\hline Layer & Compd & $\begin{array}{l}\left.\vartheta_{(\text {Prec. }}\right) \\
{\left[{ }^{\circ} \mathrm{C}\right]}\end{array}$ & $\begin{array}{l}\vartheta_{\text {(Dep. })} \\
{\left[{ }^{\circ} \mathrm{C}\right]}\end{array}$ & $\begin{array}{l}\mathrm{N}_{2} \text { flow } \\
{\left[\mathrm{mL} \mathrm{min}^{-1}\right]}\end{array}$ & $\begin{array}{l}\text { Pressure } \\
\text { [mbar] }\end{array}$ & $\begin{array}{l}\text { Dep. } \\
\text { time } \\
{[\mathrm{min}]}\end{array}$ & $\begin{array}{l}\text { Layer } \\
\text { thickness } \\
\text { [nm] }\end{array}$ \\
\hline A & $4 a$ & 130 & 400 & 50 & 0.8 & 60 & 40 \\
\hline B & $4 b$ & 130 & 400 & 50 & 0.8 & 60 & 40 \\
\hline C & 4c & 135 & 400 & 50 & 0.8 & 60 & 25 \\
\hline D & 4d & 125 & 400 & 50 & 0.8 & 60 & 25 \\
\hline E & $4 e$ & 135 & 400 & 50 & 0.8 & 60 & 55 \\
\hline $\mathrm{F}$ & 4f & 130 & 400 & 50 & 0.8 & 60 & 65 \\
\hline G & $4 e$ & 130 & 350 & 30 & 0.6 & 60 & 25 \\
\hline $\mathrm{H}^{b}$ & $4 e$ & 120 & 400 & 50 & 0.8 & 30 & 10 \\
\hline
\end{tabular}

${ }^{a}$ Determined by cross-sectional SEM images. ${ }^{b}$ Deposited on a trench patterned $\mathrm{SiO}_{2}$ wafer. 

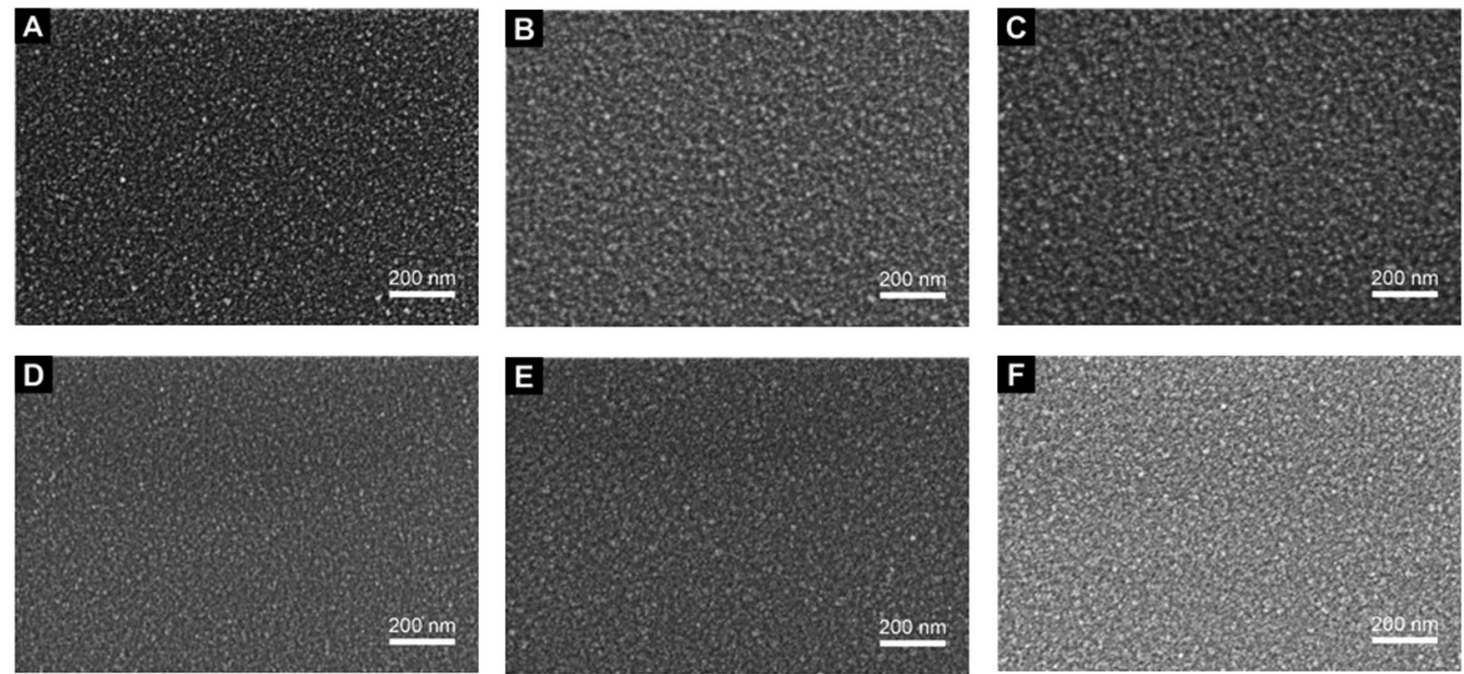

Fig. 4 SEM images (magnification: $80000 \times$ ) of ruthenium films A-F deposited on $\mathrm{SiO}_{2}$ using the parameters given in Table 2. A: $4 \mathbf{a}$, B: 4 b, C: $\mathbf{4 c}$, D: $\mathbf{4 d}$, $E: \mathbf{4 e}, F: \mathbf{4 f}$

The step coverage property of ruthenium precursor $\mathbf{4 e}$ was investigated by the deposition on a trench patterned $\mathrm{Si} / \mathrm{SiO}_{2}$ wafer with an aspect ratio of 2.5 (ESI $\dagger$ ). ${ }^{35}$ The SEM images reveal that a complete and conformal coverage of the patterned substrate was achieved for the deposition of ruthenium complex $4 \mathbf{e}$ (layer $\mathrm{H}$, Table 2).

In order to study the surface roughness, the deposited ruthenium films A-F were examined by atomic force microscopy (AFM). A representative AFM image of a ruthenium film obtained from complex $4 \mathrm{e}$ is depicted in Fig. 5 . In comparison, the AFM images taken for all samples show very similar structures with only minor variations in height. In all cases, the resulting layer topography is very homogeneous and characterized by wellinterconnected globular grains. However, the consistent bead-like shapes indicate that the actual structures are too small to be accurately imaged using a standard AFM tip. Nevertheless, all RMS (root mean square) roughness values in this study were

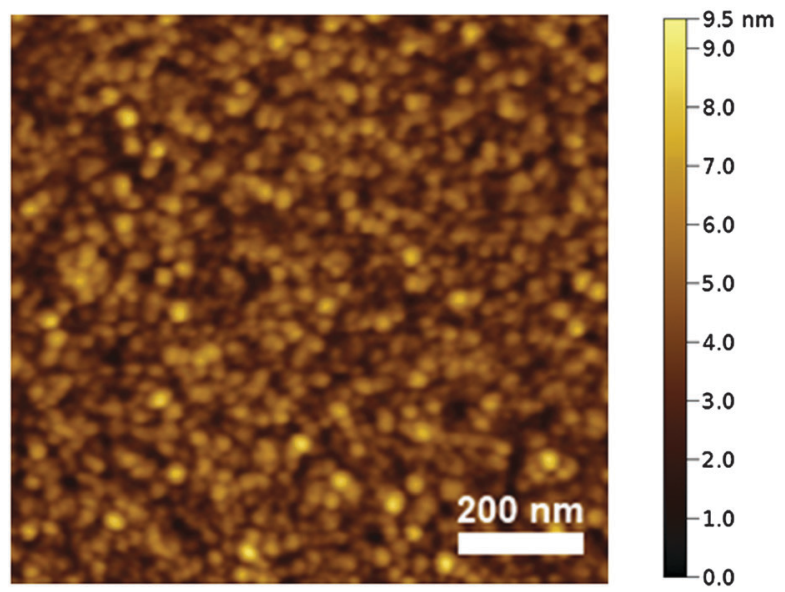

Fig. 5 AFM height image of layer $E$ (Table 2) deposited from 4 e. The RMS roughness value is $0.9 \mathrm{~nm}$. found to be in the range of $1.0 \mathrm{~nm}$ (Table 3), corresponding to relatively smoother surfaces than previously reported. ${ }^{36-40}$

The film composition was analyzed by energy-dispersive X-ray spectroscopy (EDX) and X-ray photoelectron spectroscopy (XPS). In EDX spectroscopic measurements, the presence of the characteristic pattern of ruthenium was found in all samples (ESI $\dagger$ ). In addition, the presence of phosphorus, silicon, oxygen and carbon was detected. Silicon and parts of oxygen originate from the applied $\mathrm{Si} / \mathrm{SiO}_{2}$ substrate. Hence, the intensity of these signals decreases by reducing the energy of the electron beam (penetration depth) and increasing the film thickness.

In order to determine the film composition without penetration of the $\mathrm{Si} / \mathrm{SiO}_{2}$ wafer surface sensitive XPS measurements were carried out. Thereby no silicon could be detected. Instead the presence of ruthenium, phosphorus, carbon and oxygen was confirmed. It is also necessary to note that layer F (Table 2) is fluorine-free as no fluorine could be detected by EDX or XPS analyses. In liner materials, the absence of fluorine is of particular importance as it may penetrate into the oxide layer and hence lead to device damaging. ${ }^{41}$

XPS measurements were performed on the surface as well as in the layer after argon ion sputtering $(4.0 \mathrm{keV} ; 330 \mathrm{~s})$ with the intention to remove surface contaminations (Fig. 6). The elemental contributions are summarized in Table 4 . Due to the spin-orbit

Table 3 Film properties of Ru layers A-F from Table 2

\begin{tabular}{llll}
\hline Layer & Compd & $\begin{array}{l}\text { Layer thickness } \\
{[\mathrm{nm}]}\end{array}$ & $\begin{array}{l}\text { rms roughness }^{a} \\
{[\mathrm{~nm}]}\end{array}$ \\
\hline A & $\mathbf{4 a}$ & 45 & 1.0 \\
B & $\mathbf{4 b}$ & 40 & 0.9 \\
C & $\mathbf{4 c}$ & 25 & 0.8 \\
D & $\mathbf{4 d}$ & 30 & 0.8 \\
E & $\mathbf{4 e}$ & 55 & 0.9 \\
F & $\mathbf{4 f}$ & 65 & 0.7
\end{tabular}

${ }^{a}$ Measured by AFM. 

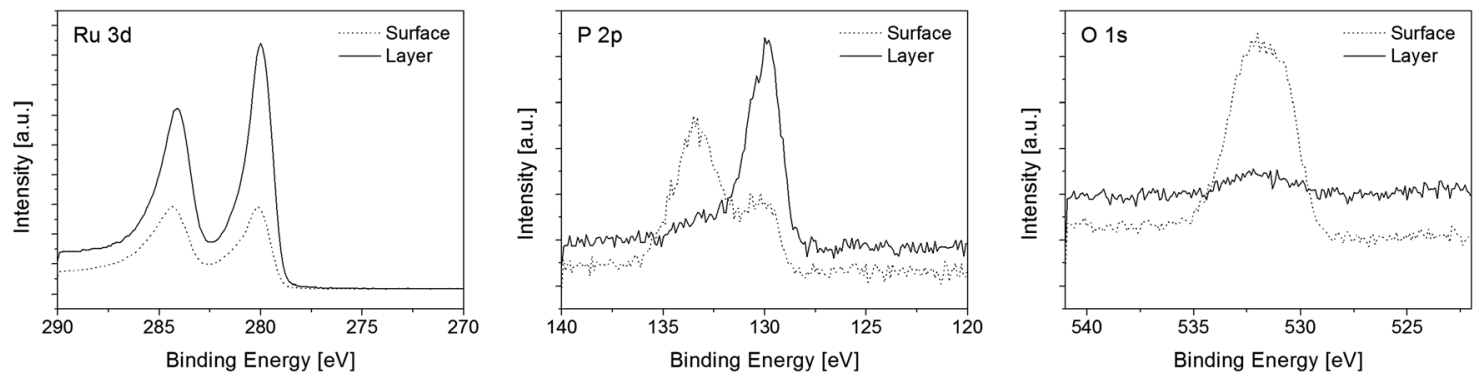

Fig. 6 Detailed XPS spectra of the surface and layer composition of film E; Ru 3d (left), P 2p (middle), O 1s (right).

interaction two peaks for $\mathrm{Ru} 3 \mathrm{~d}$ were found $\left(\mathrm{Ru} 3 \mathrm{~d}_{3 / 2}\right.$ at $284.2 \mathrm{eV}$ and $\mathrm{Ru} 3 \mathrm{~d}_{5 / 2}$ at $280.0 \mathrm{eV}$ ), which are in agreement with XPS binding energy databases and correspond to metallic ruthenium. ${ }^{42}$ These ruthenium peaks overlap with the $\mathrm{C}$ 1s peak at $284.5 \mathrm{eV},^{43}$ preventing the direct measurability of the carbon content. ${ }^{44}$ To determine the carbon content a peak deconvolution was carried out. The applied deconvolution parameters regarding the peak shape and peak position are summarized in the ESI. $\dagger$ For all peaks a maximum full width at half maximum (FWHM) of $2.0 \mathrm{eV}$ was allowed. Furthermore, the area ratio of both metallic and non-metallic $\mathrm{Ru} 3 \mathrm{~d}_{3 / 2}$ and $\mathrm{Ru} 3 \mathrm{~d}_{5 / 2}$ peaks was fixed to $2: 3$ and the peak separation between $3 \mathrm{~d}_{3 / 2}$ and $3 \mathrm{~d}_{5 / 2}$ to $4.2 \mathrm{eV}$. ${ }^{45}$ For the identification of peak splitting with respect to $\mathrm{P} 2 \mathrm{p}$ and $\mathrm{O}$ 1s a Gaussian-Lorentzian peak shape was applied with a Lorentzian contribution of $25 \%$.

The comparison of the surface and film composition reveals that the ruthenium and phosphorus contents increase in all layers, whereas the carbon and oxygen contents decrease. In general, the layers themselves consist of approximately 48-58 mol\% ruthenium, 11-16 mol\% phosphorus, 7-31 mol\% carbon and 4-29 mol\% oxygen (Table 4). The nature of the ruthenium exhibits that beside metallic species ruthenium oxide compounds are also present which can be attributed to $\mathrm{RuO}_{2}\left(\mathrm{RuO}_{2} 3 \mathrm{~d}_{5 / 2}\right.$ at $281.0 \mathrm{eV}) .{ }^{43}$ The $\mathrm{RuO}_{2}$ in the surface layer most probably arises mainly from oxygen adsorption and surface oxidation of ruthenium during the transport of the sample from the CVD reactor to the vacuum system for XPS analysis. This estimation is supported by the observation that the oxygen content clearly decreases during sputtering and the films B-F then only contain 4-7 mol\% oxygen. Since the deposition was carried out in an inert gas atmosphere, the remaining oxygen most likely originates from the dissociation of the carboxylate or CO ligands. ${ }^{46,47}$ For the phosphorus content

Table 4 Elemental contribution of layers A-F from Table 2

\begin{tabular}{|c|c|c|c|c|c|c|c|c|}
\hline \multirow[b]{2}{*}{ Layer } & \multicolumn{4}{|c|}{ Surface composition [mol\%] } & \multicolumn{4}{|c|}{ Layer composition $^{a}[\mathrm{~mol} \%]$} \\
\hline & $\mathrm{Ru} 3 \mathrm{~d}$ & P 2p & C 1s & O $1 \mathrm{~s}$ & $\mathrm{Ru} 3 \mathrm{~d}$ & P 2p & C 1s & O $1 \mathrm{~s}$ \\
\hline A & 12.4 & 9.8 & 36.3 & 41.5 & 48.0 & 16.1 & 6.9 & 29.0 \\
\hline B & 15.8 & 10.0 & 37.7 & 36.5 & 53.9 & 12.5 & 29.1 & 4.5 \\
\hline C & 17.1 & 33.3 & 38.9 & 10.7 & 51.9 & 12.9 & 29.6 & 5.6 \\
\hline $\mathrm{D}$ & 15.1 & 9.7 & 40.5 & 34.7 & 49.7 & 13.5 & 31.2 & 5.6 \\
\hline $\mathrm{E}$ & 17.6 & 11.0 & 37.1 & 34.3 & 58.1 & 11.8 & 25.5 & 4.6 \\
\hline F & 17.1 & 9.2 & 46.2 & 27.5 & 53.6 & 10.8 & 31.5 & 4.1 \\
\hline
\end{tabular}

${ }^{a}$ After argon ion sputtering ( $\left.330 \mathrm{~s}, 4.0 \mathrm{keV}\right)$. different species in the layers compared to the surface were found (Fig. 6). On the film surface signals, which can be assigned to $\mathrm{P}(\mathrm{III})$ of undecomposed phosphine ligands (P 2p at $130.2 \mathrm{eV})^{42}$ and $\mathrm{P}(\mathrm{v})$ of phosphine oxide (P 2p at $133.3 \mathrm{eV})^{48,49}$ were observed. However, within the layers only one signal was monitored, which refers to elemental phosphorus (P 2p at $129.9 \mathrm{eV}) .{ }^{42}$ Due to the small energy difference of $\mathrm{P}(0)$ and $\mathrm{P}(\mathrm{III})$ species of $0.3 \mathrm{eV}$, it cannot be excluded that $\mathrm{P}(\mathrm{III})$ is present within the layers. ${ }^{42}$ The carbon impurities arise from the catalytic properties of the ruthenium surface, which lead to $\mathrm{C}-\mathrm{H}$ and $\mathrm{C}-\mathrm{C}$ activation of the adsorbed ligands resulting in the incorporation of elemental carbon. ${ }^{50,51}$

To evaluate the crystallinity of the deposited films X-ray powder diffraction (XRPD) measurements were performed (Fig. S6, ESI $\dagger$ ). The XRPD pattern shows some diffraction arising from the $\mathrm{Si} / \mathrm{SiO}_{2}$ substrate. As no peaks originating from the $\mathrm{Ru}(\mathrm{P})$ film could be determined the layers are most probably amorphous.

\section{Conclusion}

Ruthenium complexes $\mathrm{Ru}(\mathrm{CO})_{2}\left(\mathrm{P}(n-\mathrm{Bu})_{3}\right)_{2}\left(\mathrm{O}_{2} \mathrm{CR}\right)_{2}(\mathbf{4 a}, \mathrm{R}=\mathrm{Me} ; \mathbf{4 b}$, $\mathrm{R}=\mathrm{Et} ; \mathbf{4 c}, \mathrm{R}=\mathrm{i}-\mathrm{Pr} ; \mathbf{4 d}, \mathrm{R}=t$ - $\mathrm{Bu} ; \mathbf{4 e}, \mathrm{R}=\mathrm{CH}_{2} \mathrm{OCH}_{3} ; \mathbf{4 f}, \mathrm{R}=\mathrm{CF}_{3} ; \mathbf{4 g}$, $\mathrm{R}=\mathrm{CF}_{2} \mathrm{CF}_{3}$ ) were successfully applied as single-source precursors for the deposition of phosphorus-doped ruthenium layers by the CVD process. The ruthenium compounds were synthesized by a "one-pot" synthetic methodology reacting $\mathrm{Ru}_{3}(\mathrm{CO})_{12}$ with $\mathrm{P}(n-\mathrm{Bu})_{3}$ and the respective carboxylic acid. All precursors are stable against air or moisture and allow a liquid precursor delivery in a continuous CVD process due to their low melting points. Additional advantages of $\mathbf{4 a}-\mathbf{g}$ are that their thermal properties including melting points, vapor pressures and decomposition behaviors can easily be modified by the introduction of different carboxylate ligands. Deposition was carried out in a cold-wall CVD reactor at deposition temperatures between 350 and $400{ }^{\circ} \mathrm{C}$ in an inert gas atmosphere without the need of any additional phosphorus source or reactive gas. The highest growth rates were observed for $\mathbf{4 e}$ and 4f, which also exhibited the highest vapor pressures. All received films were dense and conformal as proven by SEM images and were also uniform in the deposition on patterned wafers with an aspect ratio of 2.5. Furthermore, they possess a very low surface roughness with an RMS value of approximately $1.0 \mathrm{~nm}$ as determined by AFM. The elemental composition of the layers was analyzed by EDX and XPS measurements.

In conclusion, ruthenium complexes $\mathbf{4 a}-\mathbf{f}$ show promising properties for the fabrication of homogeneous and conformal 
phosphorus-doped ruthenium layers, as all complexes are easy to synthesize, stable in air and do not need a reactive gas or an additional phosphorus source during deposition. The relatively high phosphorus contents of up to $16 \mathrm{~mol} \%$ make the resulting layers attractive as single material liners for copper interconnects in the damascene process. In the future, the application of phosphine ligands with shorter alkyl chains will lead to significantly decreased carbon contents. Investigations concerning this matter are currently in progress.

\section{Acknowledgements}

This work was supported by the Fonds der Chemischen Industrie. J. J. and M. K. thank the Fonds der Chemischen Industrie for Chemiefonds fellowships. We thank Heraeus for the generous gift of $\mathrm{Ru}_{3}(\mathrm{CO})_{12}$. In addition, we acknowledge Cornelia Kowol and Dirk Rittrich for performing the SEM/EDX measurements, Lutz Mertens and Prof. Michael Mehring for the XRPD analyses and Prof. Robert Magerle for permitting us to perform the AFM studies on his devices.

\section{References}

1 A. E. Kaloyeros, E. T. Eisenbraun, K. Dunn and O. van der Straten, Chem. Eng. Commun., 2011, 198, 1453-1481.

2 A. Tuchscherer, C. Georgi, N. Roth, D. Schaarschmidt, T. Rüffer, T. Waechtler, S. E. Schulz, S. Oswald, T. Gessner and H. Lang, Eur. J. Inorg. Chem., 2012, 4867-4876.

3 O.-K. Kwon, S.-H. Kwon, H.-S. Park and S.-W. Kang, J. Electrochem. Soc., 2004, 151, C753-C756.

4 O.-K. Kwon, J.-H. Kim, H.-S. Park and S.-W. Kang, J. Electrochem. Soc., 2004, 151, G109-G112.

5 D. Josell, D. Wheeler, C. Witt and T. P. Moffat, Electrochem. Solid-State Lett., 2003, 6, C143-C145.

6 S.-H. Choi, T. Cheon, S.-H. Kim, D.-H. Kang, G.-S. Park and S. Kim, J. Electrochem. Soc., 2011, 158, D351-D356.

7 T. Cheon, S.-H. Choi, S.-H. Kim and D.-H. Kang, Electrochem. Solid-State Lett., 2011, 14, D57-D61.

8 D.-C. Perng, J.-B. Yeh and K.-C. Hsu, Appl. Surf. Sci., 2008, 254, 6059-6062.

9 J.-J. Tan, X.-P. Qu, Q. Xie, Y. Zhou and G.-P. Ru, Thin Solid Films, 2006, 504, 231-234.

10 T. P. Moffat, M. Walker, P. J. Chen, J. E. Bonevich, W. F. Egelhoff, L. Richter, C. Witt, T. Aaltonen, M. Ritala, M. Leskelä and D. Josell, J. Electrochem. Soc., 2006, 153, C37-C50.

11 J. Shin, A. Waheed, K. Agapiou, W. A. Winkenwerder, H.-W. Kim, R. A. Jones, G. S. Hwang and J. G. Ekerdt, J. Am. Chem. Soc., 2006, 128, 16510-16511.

12 T. N. Arunagiri, Y. Zhang, O. Chyan, M. El-Bouanani, M. J. Kim, K. H. Chen, C. T. Wu and L. C. Chen, Appl. Phys. Lett., 2005, 86, 83104.

13 L. B. Henderson and J. G. Ekerdt, Thin Solid Films, 2009, 517, 1645-1649.

14 D.-C. Perng, K.-C. Hsu, S.-W. Tsai and J.-B. Yeh, Microelectron. Eng., 2010, 87, 365-369.
15 J. Shin, H.-W. Kim, G. S. Hwang and J. G. Ekerdt, Surf. Coat. Technol., 2007, 201, 9256-9259.

16 J. Shin, H.-W. Kim, K. Agapiou, R. A. Jones, G. S. Hwang and J. G. Ekerdt, J. Vac. Sci. Technol., A, 2008, 26, 974-979.

17 J. Shin, A. Waheed, W. A. Winkenwerder, H.-W. Kim, K. Agapiou, R. A. Jones, G. S. Hwang and J. G. Ekerdt, Thin Solid Films, 2007, 515, 5298-5307.

18 D. E. Bost and J. G. Ekerdt, Thin Solid Films, 2014, 558, 160-164.

19 H. Lang and S. Dietrich, in Comprehensive Inorganic Chemistry II, ed. J. R. Poeppelmeier, Elsevier, Amsterdam, 2013.

20 T. T. Kodas and M. J. Hampden-Smith, The Chemistry of Metal CVD, Wiley-VCH, Weinheim, 1994.

21 J. Jeschke, C. Georgi and H. Lang, Patent application, DE102014205342A1, 2014.

22 G. M. Sheldrick, SHELXL - Program for Crystal Structure Refinement, Universität Göttingen, 1997.

23 G. M. Sheldrick, Acta Crystallogr., Sect. A: Found. Crystallogr., 2008, 64, 112-122.

24 L. J. Farrugia, J. Appl. Crystallogr., 2012, 45, 849-854.

25 M. Bianchi, P. Frediani, U. Matteoli, G. Menchi, F. Piacenti and G. Petrucci, J. Organomet. Chem., 1983, 259, 207-214.

26 J. Jeschke, C. Gäbler, M. Korb, T. Rüffer and H. Lang, Eur. J. Inorg. Chem., 2015, 2939-2947.

27 J. Jeschke, M. Korb, T. Rüffer, C. Gäbler and H. Lang, Adv. Synth. Catal., 2015, 357, 4069-4081.

28 H. G. Metzinger, Org. Magn. Reson., 1971, 3, 485-494.

29 R. K. Harris, Can. J. Chem., 1964, 42, 2275-2281.

30 B. F. G. Johnson, R. D. Johnston, J. Lewis and I. G. Williams, J. Chem. Soc. A, 1971, 689-691.

31 G. B. Deacon and R. J. Phillips, Coord. Chem. Rev., 1980, 33, 227-250.

32 C. Georgi, A. Hildebrandt, T. Waechtler, S. E. Schulz, T. Gessner and H. Lang, J. Mater. Chem. C, 2014, 2, 4676-4682.

33 C. Georgi, M. Hapke, I. Thiel, A. Hildebrandt, T. Waechtler, S. E. Schulz and H. Lang, Thin Solid Films, 2015, 578, 180-184.

34 F.-J. Lee, Y. Chi, C.-S. Liu, P.-F. Hsu, T.-Y. Chou, S.-M. Peng and G.-H. Lee, Chem. Vap. Deposition, 2001, 7, 99-101.

35 G. B. Raupp and T. S. Cale, Chem. Mater., 1989, 1, 207-214.

36 J. J. Kim, M. S. Kim and D. Y. Yoon, Chem. Vap. Deposition, 2003, 9, 105-109.

37 I. Jipa, K. Danova, N. Popovska, M. A. Siddiqi, R. A. Siddiqui, B. Atakan, T. Cremer, F. Maier, H. Marbach, H.-P. Steinrück, F. W. Heinemann and U. Zenneck, J. Mater. Chem., 2011, 21, 3014-3024.

38 K. M. Thom and J. G. Ekerdt, Thin Solid Films, 2009, 518, 36-42.

39 J. J. Kim, D. H. Jung, M. S. Kim, S. H. Kim and D. Y. Yoon, Thin Solid Films, 2002, 409, 28-32.

40 A. Schneider, N. Popovska, I. Jipa, B. Atakan, M.-A. Siddiqi, R. Siddiqui and U. Zenneck, Chem. Vap. Deposition, 2007, 13, 389-395.

41 K. Chang, I. Deng and H. Lin, J. Electrochem. Soc., 1999, 146, 3092-3096. 
42 NIST X-ray Photoelectron Spectroscopy Database, Version 4.1., National Institute of Standards Technology, Gaithersburg, USA, 2012.

43 H. Y. H. Chan, C. G. Takoudis and M. J. Weaver, J. Catal., 1997, 172, 336-345.

44 A. Iembo, F. Fuso, E. Arimondo, C. Ciofi, G. Pennelli, G. M. Curró, F. Neri and M. Allegrini, J. Mater. Res., 1997, 12, 1433-1436.

45 J. Y. Shen, A. Adnot and S. Kaliaguine, Appl. Surf. Sci., 1991, 51, 47-60.

46 E. Shincho, C. Egawa, S. Naito and K. Tamaru, Surf. Sci., 1985, 149, 1-16.
47 D. E. Starr and H. Bluhm, Surf. Sci., 2013, 608, 241-248.

48 C. A. Strydom and H. J. Strydom, Inorg. Chim. Acta, 1989, 159, 191-195.

49 A. Ghicov, H. Tsuchiya, J. M. Macak and P. Schmuki, Electrochem. Commun., 2005, 7, 505-509.

50 A. Schneider, N. Popovska, F. Holzmann, H. Gerhard, C. Topf and U. Zenneck, Chem. Vap. Deposition, 2005, 11, 99-105.

51 I. Jipa, M. A. Siddiqi, R. A. Siddiqui, B. Atakan, H. Marbach, T. Cremer, F. Maier, H.-P. Steinrück, K. Danova, N. Popovska, F. W. Heinemann and U. Zenneck, Chem. Vap. Deposition, 2011, 17, 15-21. 